\title{
Usefulness of the Wisconsin and CaPTHUS indices for predicting multiglandular disease in patients with primary hyperparathyroidism in a southern European population
}

\author{
Mario Serradilla-Martín ${ }^{1} \wedge$, Ana Palomares-Cano ${ }^{\wedge} \wedge$, Miguel Cantalejo-Díaz $^{2} \wedge$, \\ Mónica Mogollón-González ${ }^{3 \wedge}$, Esther Brea-Gómez ${ }^{3 \wedge}$, Nuria Victoria Muñoz-Pérez ${ }^{3 \wedge}$, \\ Juan Ignacio Arcelus-Martínez ${ }^{3 \wedge}$, Jesús María Villar-del-Moral ${ }^{3 \wedge}$ \\ ${ }^{1}$ Instituto de Investigación Sanitaria Aragón, Department of Surgery, Miguel Servet University Hospital, Zaragoza, Spain; ${ }^{2}$ Department of Surgery, \\ Miguel Servet University Hospital, Zaragoza, Spain; ${ }^{3}$ Department of Surgery, Virgen de las Nieves University Hospital, Granada, Spain \\ Contributions: (I) Conception and design: M Serradilla-Martín, JI Arcelus-Martínez, JM Villar-del-Moral; (II) Administrative support: M Serradilla- \\ Martín; (III) Provision of study materials or patients: JI Arcelus-Martínez, JM Villar-del-Moral, NV Muñoz-Pérez; (IV) Collection and assembly \\ of data: M Serradilla-Martín, A Palomares-Cano, M Cantalejo-Díaz, M Mogollón-González, E Brea-Gómez, NV Muñoz-Pérez; (V) Data analysis \\ and interpretation: M Serradilla-Martín, JI Arcelus-Martínez, JM Villar-del-Moral; (VI) Manuscript writing: All authors; (VII) Final approval of \\ manuscript: All authors. \\ Correspondence to: Mario Serradilla-Martín. Department of Surgery, Miguel Servet University Hospital, Paseo Isabel La Católica, 1-3, 50009 \\ Zaragoza, Spain. Email: marioserradilla@hotmail.com.
}

Background: Focused parathyroidectomy is a safe technique for the treatment of primary hyperparathyroidism. The CaPTHUS score and the Wisconsin index are preoperative diagnostic tools designed to distinguish between single- and multigland disease. The aim of the study is to evaluate the usefulness of these models for predicting multiglandular disease in a European population.

Methods: Retrospective review of a database of patients operated upon for primary hyperparathyroidism at a referral center. The sensitivity, specificity, positive and negative predictive values, and reliability of both scores for the prediction of multiglandular disease, were calculated. Receiver operating characteristic (ROC) curves were constructed to assess the sensitivity and specificity of CaPTHUS score and Wisconsin Index for predicting single-gland disease. A level of $\mathrm{P}<0.05$ was accepted as significant.

Results: Two hundred and eighty-one patients who underwent successful surgery from January 2001 to December 2018 were included. Single-gland disease was detected in $92.5 \%$, and $73.7 \%$ had a CaPTHUS score of $\geq 3$. The sensitivity, specificity, positive and negative predictive values of this model for predicting singlegland disease with a score of $\geq 3$ were $76.9 \%, 66.7 \%, 96.6 \%$, and $18.9 \%$ respectively. The area under the curve value of the CaPTHUS score for predicting single-gland disease was 0.74 . A Wisconsin Index $>2,000$ and an excised gland weight above one gram presented a positive predictive value for single-gland disease of $92.5 \%$.

Conclusions: Despite the good performance of both scales, the established cut-off points did not definitively rule out parathyroid multiglandular disease in our population. In cases with a minimal suspicion of this condition, additional intraoperative techniques must be used, or bilateral neck explorations should be performed.

Keywords: Primary hyperparathyroidism; multigland disease (MGD); Wisconsin index (WIN); CaPTHUS score; selective parathyroidectomy (SP)

\footnotetext{
^ ORCID: Mario Serradilla-Martín, 0000-0002-2466-0711; Ana Palomares-Cano, 0000-0003-1686-3356; Miguel Cantalejo-Díaz, 00000002-5123-4849; Mónica Mogollón-González, 0000-0001-7684-5067; Esther Brea-Pérez, 0000-0001-6318-9582; Nuria Victoria MuñozPérez, 0000-0002-6754-8535; Juan Ignacio Arcelus-Martínez, 0000-0003-2283-3233; Jesús María Villar-del Moral, 0000-0001-6831-6739.
} 
Submitted Nov 29, 2020. Accepted for publication Feb 04, 2021.

doi: $10.21037 /$ gs-20-857

View this article at: http://dx.doi.org/10.21037/gs-20-857

\section{Introduction}

Primary hyperparathyroidism (PHPT) is a calcium metabolism disorder derived from excessive secretion of parathyroid hormone (PTH), leading to abnormally high levels of serum calcium (1-3). It is the most common cause of hypercalcemia in outpatients (2-4). The prevalence is 1 per 1,000 in men and 2-3 per 1,000 in women, a rate that rises to $1 \%$ in postmenopausal women. The difference between genders is more evident with increasing age (2,5-7). Most PHPTs are caused by a single adenoma ( $80-85 \%)$. However, the involvement of more than one gland is not uncommon, in the form of parathyroid hyperplasia (14-20\%) or multiple adenomas (3\%) (1,8-10).

The possible presence of multigland disease (MGD) is a major concern in PHPT surgery. Classically, the standard surgery consisted of exploring all the glands and removing the pathological ones; today, the emergence and improvement of preoperative localization tests and intraoperative adjuncts have established selective parathyroidectomy (SP) as the most common surgical strategy. This technique obtains postoperative normocalcemia rates similar to bilateral neck exploration (BNE), and reduces operative time, hospital stay, risk of complications and healthcare costs (11-14). SP targets a specific pathological gland based on its preoperative identification and on the intraoperative use of complementary tools $(15,16)$, with intraoperative PTH measurement (ioPTH) being the most widely applied (17).

In recent years, several different systems have been designed to preoperatively identify patterns of patients with MGD and thus avoid SP failure. One of them is the CaPTHUS scoring model, described by Kebebew et al. in 2006 (18), which has been shown to be a useful tool by its creators. Similarly, in 2013 Mazeh et al. (19) devised the Wisconsin Index (WIN) for predicting the probability of the presence of additional hyperfunctioning glands during SP.

Given the possible differences between US and European populations with regard to the clinicopathological characteristics of hyperparathyroidism (5-7), the present study was designed to evaluate the usefulness of WIN and CaPTHUS indices for predicting MGD in patients with PHPT in a European population.

We present the following article in accordance with the STARD reporting checklist (available at http://dx.doi. org/10.21037/gs-20-857).

\section{Methods}

A retrospective review was performed of a cohort of patients who had undergone PHPT surgery consecutively at an Endocrine Surgery Unit in southern Europe. Data were obtained from a prospectively maintained database. Demographic and biochemical variables, imaging studies, surgical approach, operative findings, ioPTH results, histology, weight of the excised glands, and clinical and biochemical follow-up data were analysed. An analysis of the average preoperative calcium and PTH values was performed to evaluate differences in the surgical indication.

The following exclusion criteria were applied: lack of preoperative calcium and/or PTH determination, no preoperative imaging workup, lack of relevant perioperative variables, loss to follow-up, cervicotomy without glandular resection, parathyroid incidentalomas excised during thyroidectomies, diagnosis of parathyroid cancer, and nonsuccessful surgery (persistent postoperative hipercalcemia).

Cervical Doppler ultrasound and technetium$99 \mathrm{~m}$ Sestamibi scintigraphy were used as preoperative localization workup. Patients with concordant studies suggesting single-gland disease (SGD) were treated with SP supported by ioPTH, evaluated using Miami criteria (16), available from the beginning of the study period. In cases with non-concordant results, but both tests suggested location on the same side, a unilateral neck exploration was proposed. BNE was reserved for doubly negative, discordant studies or those suggestive of MGD. Eucalcemia maintained at least six months after surgery was regarded as indicating cure.

The patients were divided into two groups: those with SGD (single adenoma) and those with MGD (double adenoma or multiglandular hyperplasia). The CaPTHUS score was calculated, as defined in the original article, awarding patients one point for each of the five predictive factors met: preoperative $\mathrm{Ca} \geq 12 \mathrm{mg} / \mathrm{dL}$, intact PTH $\geq 2$ times the upper limit of normality, ultrasound and scintigraphy results suggestive of SGD, and concordance between the two techniques. As in the original study (18), 


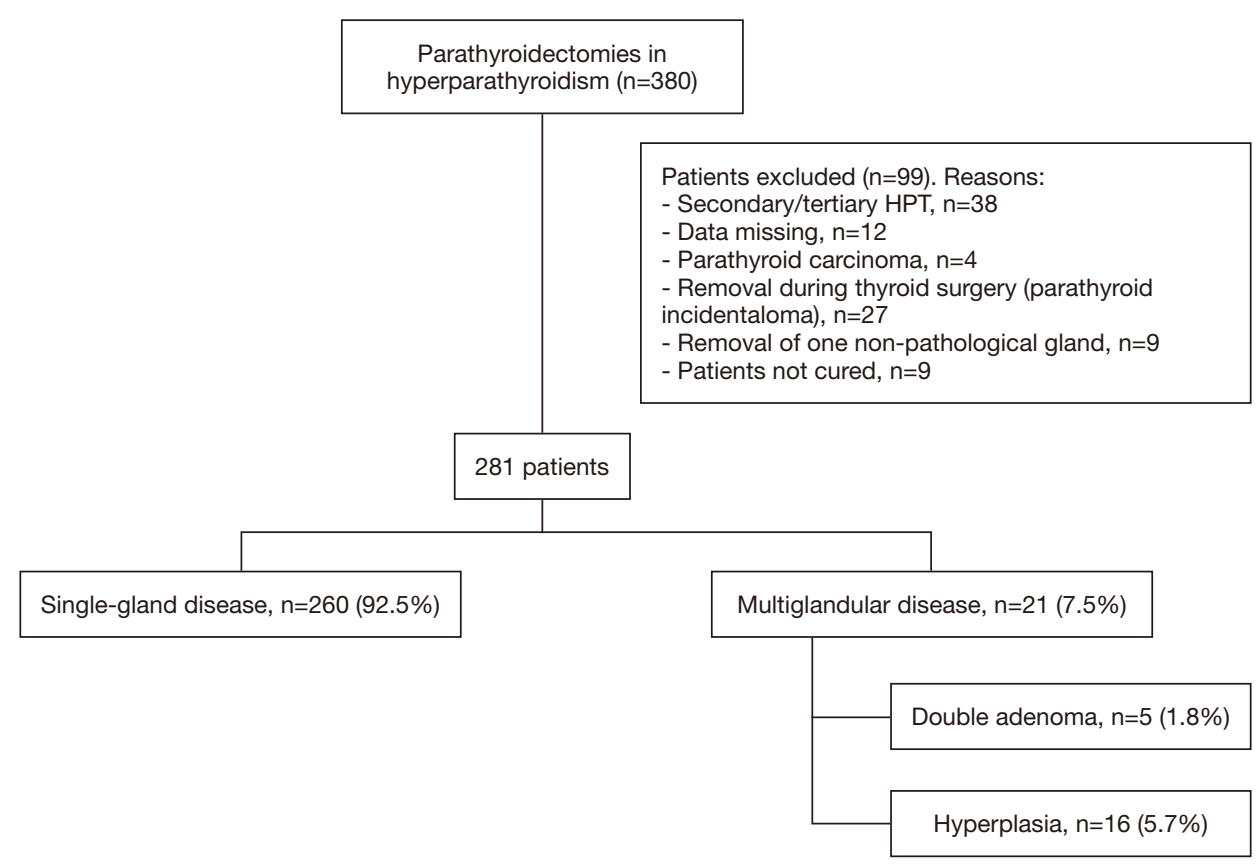

Figure 1 Flow chart of the study.

a score $\geq 3$ was used as a cut-off point for predicting SGD. Unlike calcemia, PTH normal values are defined by each laboratory.

Likewise, preoperative serum calcium and PTH values were used to determine the WIN. This was obtained by multiplying the two values. The cohort was divided into three groups including low, medium or high probability of having MGD based on the score obtained when comparing these data with the weight of all the pathologic excised glands (19).

\section{Statistical analysis}

Categorical variables are presented as frequencies and percentages. Continuous variables adjusted to normality are described by means and standard deviations, otherwise with median and interquartile range (IR). The sensitivity, specificity, negative predictive value (NPV), positive predictive value (PPV) and global accuracy of both scores for predicting MGD were calculated. Comparisons between categorical variables were made using chi-square tests with Yates correction. Differences between 2 continuous variables were assessed using Student's and Mann-Whitney $\mathrm{U}$ tests for normal and non-parametric distributions respectively. The non-parametric Kruskal-Wallis $\mathrm{H}$ test was used for comparing 3 variables. Spearman's rank correlation measured the strength of association between variables of interest. Receiver operating characteristic (ROC) curves were constructed to assess the sensitivity and specificity of the CaPTHUS and WIN scores to predict SGD. The data were analysed using $\mathrm{R}$ version 3.1.3. (http://www.r-project. org). Values of $\mathrm{P}<0.05$ were considered significant.

\section{Ethical aspects}

Patients' informed consent was not required since this was a retrospective and observational study that did not entail any risk. The study was conducted in accordance with the Declaration of Helsinki (as revised in 2013). The study was approved by the regional ethics committee of Granada (PI19/127).

\section{Results}

From January 2001 to December 2018, 380 patients underwent parathyroidectomy for hyperparathyroidism at our Unit. Of these, 337 underwent PHPT surgery, of whom 281 met the study inclusion criteria (Figure 1). Fifty-four were men (19.2\%) and 227 women $(80.8 \%)$, with a mean age of 58.6 years $( \pm 12.9)$. Of these, 260 presented SGD (92.5\%) and 21 MGD (7.5\%).

Demographic data and baseline characteristics are shown in Table 1. The number of patients diagnosed with hereditary PHPT in the MGD group was significantly higher than in 
Table 1 Demographic data and baseline characteristics of the study cohort

\begin{tabular}{|c|c|c|c|c|}
\hline Clinical characteristics & Single-gland disease $(\mathrm{N}=260)$ & Multiglandular disease $(\mathrm{N}=21)$ & Total $(\mathrm{N}=281)$ & $\mathrm{P}$ \\
\hline Female gender & $211(81.2)$ & $16(76.2)$ & $227(80.8)$ & 0.568 \\
\hline Age (years) & $59.1(12.6)$ & $53.3(15.3)$ & $58.6(12.9)$ & 0.107 \\
\hline Calcium value (mg/dL) & $11.2(10.8-11.7)$ & $11.5(10.9-12.0)$ & $11.2(10.8-11.8)$ & 0.271 \\
\hline ioPTH complying with Milan criteria (16) & $243(93.5)$ & $21(100.0)$ & $264(94.0)$ & 0.608 \\
\hline Total weight of gland(s) removed (mg) & $765(370-1,574)$ & $1,100(320-1,740)$ & $780(370-1,640)$ & 0.264 \\
\hline
\end{tabular}

The categorical variables are expressed by the number of cases (\%) and the continuous variables by the median (interquartile ranges). Comparisons between groups were made using the $\mathrm{chi}^{2}$ test for categorical variables and the Student's $t$-test for continuous variables. PHPT, primary hyperparathyroidism; PTH, parathyroid hormone; ioPTH, intraoperative parathyroid hormone monitoring.

Table 2 Comparison of the differences between patients with SGD and MGD regarding to the factors of the CaPTHUS score

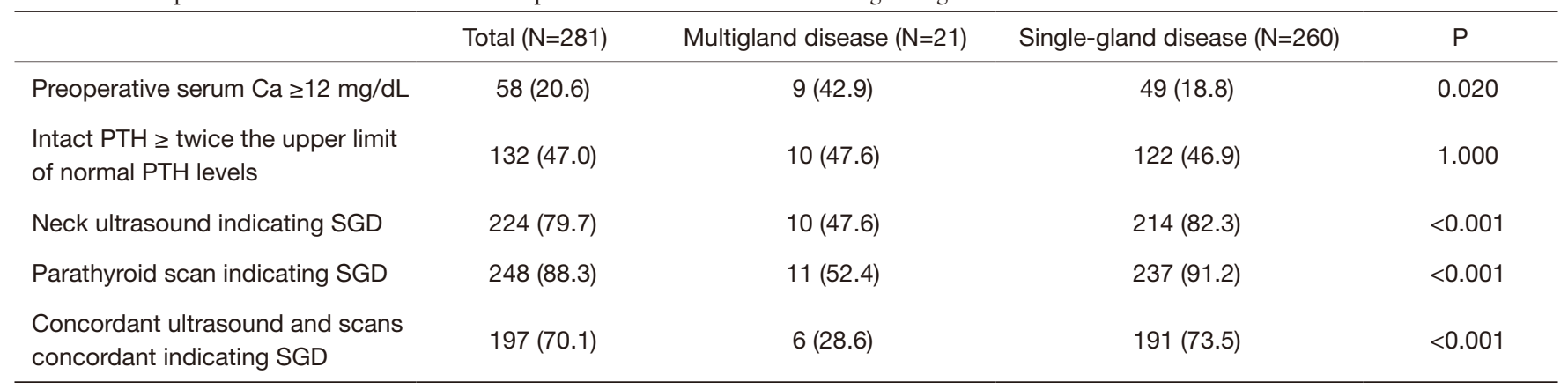

The data are expressed by the number of cases (\%). Comparisons between groups were made using the chi ${ }^{2}$ test. $\mathrm{PTH}$, parathyroid $^{2}$ hormone; SGD, single-gland disease; MGD, multigland disease.

the SGD group, all suffering a MEN I syndrome.

When the trend lines of the models that included the annual variation of calcium levels and $\mathrm{PTH}$ were adjusted, an average decrease in calcium levels of $0.57 \mathrm{mg} / \mathrm{dL}$ was observed, while the average iPTH did not vary throughout the period of study.

Sensitivity and specificity of ultrasound for the detection of SGD were $82.9 \%$ and $52.4 \%$ respectively. For scintigraphy, the corresponding figures were $91.1 \%$ (sensitivity) and $47.6 \%$ (specificity). Thirty-three patients did not show any abnormal glands. Among these patients, the final histology study revealed MGD in 10 (30.3\%): four double adenomas, and six due to multiglandular hyperplasia. BNE was performed in 113 patients (40.2\%); a more selective approach was applied in the remaining 168 (59.8\%), with $136 \mathrm{SP}$ and 32 unilateral explorations. These selective approaches were successful in $97.3 \%$ of cases. In only $70 \%$ of the patients the cervical ultrasound and technetium-99m Sestamibi scintigraphy was concordant and suggestive of SGD. In addition, $10 \%$ of the patients with PHPT had concomitant thyroid disease with surgical indication (uni or multinodular goiter). This, added to the $30 \%$ of discordant preoperative workup or suspicion of MGD justify $40.2 \%$ of BNE.

Table 2 compares the differences between SGD and MGD regarding the factors of the CaPTHUS score. The median score was 3 (IR $=2-4$ ), with $73.7 \%$ of patients scoring $\geq 3$. The percentage of patients with SGD increased with higher CaPTHUS scores, being $96.6 \%$ for scores $\geq 3(94.8 \%, 98.2 \%$ and $100 \%$ for scores of 3,4 and 5 respectively). The CaPTHUS score of our cohort is shown in Table 3. The sensitivity, specificity, PPV and NPV of this model for predicting SGD for a score $\geq 3$ were $76.9 \%$, $66.7 \%, 96.6 \%$ and $18.9 \%$ respectively.

Regarding the choice of surgical approach, patients with CaPTHUS scores of $\geq 3$ underwent BNE less often than 
Table 3 CaPTHUS scores in the whole series and in subgroups

\begin{tabular}{lccc}
\hline CaPTHUS score & Total $(\mathrm{N}=281)$ & Multigland disease $(\mathrm{N}=21)$ & Single-gland disease $(\mathrm{N}=260)$ \\
\hline 0 & $2(0.71)$ & $0(0.0)$ & $2(0.8)$ \\
1 & $33(11.7)$ & $4(19.0)$ & $29(11.2)$ \\
2 & $39(13.9)$ & $10(47.6)$ & $29(11.2)$ \\
3 & $115(40.9)$ & $6(28.6)$ & $109(41.9)$ \\
4 & $57(20.3)$ & $1(4.8)$ & $56(21.5)$ \\
5 & $35(12.5)$ & $0(0.0)$ & $35(13.5)$ \\
\hline
\end{tabular}

The data are expressed by the number of cases and percentages. Comparisons between groups were made using the chi ${ }^{2}$ test. CaPTHUS score $0-2$ and $3-5, P<0.001$.

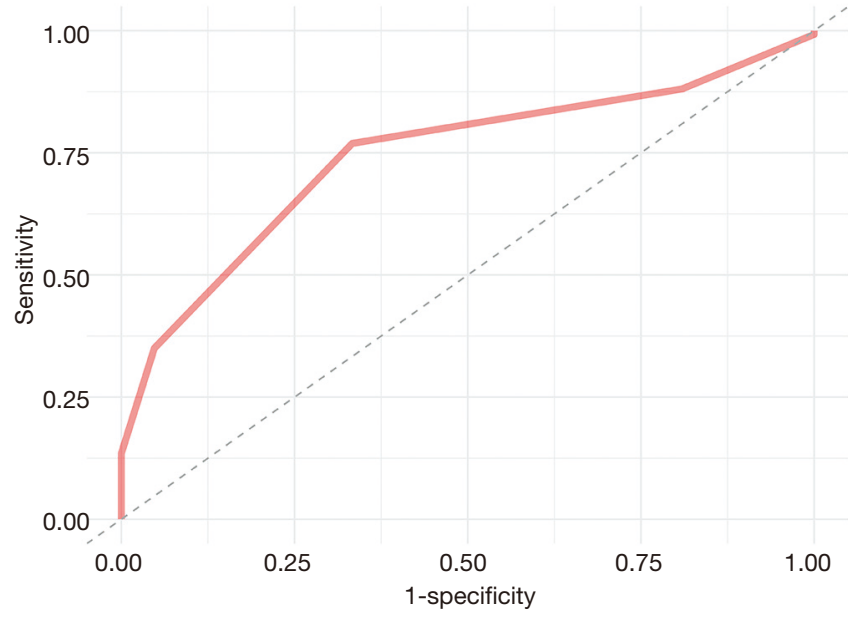

Figure 2 Receiver operating characteristic (ROC) curve of the precision of the scoring model for predicting single-gland disease (SGD), with an area under the curve (AUC) of 0.74 (95\% CI, $0.66-0.83)$.

those with lower scores $(25.1 \%$ vs. $59.4 \%, \mathrm{P}<0.001)$; the rate in the entire cohort was $40.2 \%$. Figure 2 shows the ROC curve for the CaPTHUS score predictor of SGD, with an area under the curve (AUC) value of 0.74.

The median WIN, obtained by multiplying the serum calcium and PTH values, was 1,691 (IR = 810-32,518). The scores were defined as low $(<1,300)$, medium $(1,301-2,000)$ or high $(>2,000)$. The relationship between the defined categories of calcemia, PTH and WIN with the gland weight is displayed in Table 4. The linear correlation study showed a positive relation between WIN and weight of the removed gland $(r=0.334, \mathrm{P}<0.0001)$. A score $\mathrm{WIN}>2,000$ with a weight of the excised gland above one gram had a PPV for SGD of $92.5 \%$.
Table 4 Relationship between the defined categories for calcemia, ioPTH and WIN with the weight of the excised glands

\begin{tabular}{lccc}
\hline Quantitative variables & $\mathrm{N}$ & Median $[\mathrm{IR}](\mathrm{mg})$ & $\mathrm{P}$ \\
\hline Calcemia $(\mathrm{mg} / \mathrm{dL})$ & & & $<0.001$ \\
$<11$ & 87 & $730[383-1,035]$ & \\
$11-11.5$ & 103 & $560[332-1,029]$ & \\
$>11.5$ & 91 & $1,200[552-2,855]$ & \\
ioPTH $(\mathrm{pg} / \mathrm{mL})$ & & & $<0.001$ \\
$\leq 120$ & 81 & $650[330-920]$ & \\
$120-179$ & 96 & $680[360-1,425]$ & \\
$>180$ & 104 & $1,005[530-2,630]$ & \\
WIN & & & $<0.001$ \\
$\leq 1,300$ & 84 & $650[344-920]$ & \\
$1,300-2,000$ & 93 & $700[360-1,400]$ & \\
$>2,000$ & 104 & $1,055[530-2,847]$ & \\
\hline
\end{tabular}

Comparisons between groups were made using the KruskalWallis test. ioPTH, intraoperative parathyroid hormone; WIN, Wisconsin index; IR, interquartile range.

\section{Discussion}

Preoperative identification of MGD remains a major concern in PHPT surgery. Despite the profusion of preoperative localization tests and intraoperative adjuncts, non-curative treatment is not exceptional, and some patients will suffer persistent disease due to incomplete intraoperative detection $(1,20,21)$.

In recent decades, several algorithms have been developed for ruling out MGD. The CaPTHUS score, described by Kebebew et al. in 2006 (18) and validated by Elfenbein et al. in 2015 (22), provides a good estimate of the probability of 
MGD, with a precision of almost 100\% (18). Kavanagh et al. found comparable results with a similar scoring system (17). However, Elfenbein et al. also included patients who had undergone only ultrasound or scintigraphy and showed that with a score of $\geq 3$ the PPV decreased to $91 \%$ for predicting SGD. In their hands, the lack of measuring ioPTH for patients with high CaPTHUS scores had a cure rate of $89 \%$ at 6 months, compared with $98 \%$ when this assay was performed (22).

In 2013, Mazeh et al. created the WIN, in order to predict the likelihood of the presence of additional hyperfunctioning glands during SP (19). In their results, the WIN score was closely associated with the overall weight of parathyroid disease, and proved useful for assessing the risk of leaving pathological parathyroid tissue in situ during SP. Thus, the combination of the WIN and gland weight allowed the creation of a nomogram that stratified the patients into categories of high, medium or low risk of MGD.

In 2017, Edafe et al. (23) simultaneously applied CaPTHUS and WIN to discriminate between SGD and MGD in their two-center cohort comprising 624 patients. The PPV for predicting SGD of the CaPTHUS $\geq 3$ model was $84.6 \%$. There were no significant differences in the WIN between patients with SGD and MGD ( $\mathrm{P}=0.573)$. In this study, a $W I N \geq 1,600$ and a gland weight of $\geq 1 \mathrm{~g}$ had a PPV for detecting SGD of $86.7 \%$.

Recently, other imaging methods have been used to identify hyperfunctioning glands. In 2015, Sepahdari et al. proposed a scoring model based on the findings of fourdimensional computed tomography (CT-4D) images, including variables such as largest lesion size and number of lesions, in combination with biochemical data (preoperative calcium and PTH, and WIN) (24). However, the sensitivity of CT-4D to detect MGD remained low (32-53\%), compared to its sensitivity for detecting SGD (88-93\%) (25-28).

Several recent meta-analyses have found that another innovative technique, F18-choline positron emission tomography associated with computerized axial tomography (PET/CT), has a sensitivity of $95 \%$ and a PPV of $97 \%$ for detecting pathological parathyroids in cases of PHPT (29-32). However, large multicenter studies and a cost-effectiveness analysis are needed to better define its role in this pathology (30).

In our study, median age, gender proportion, and surgical strategy used were similar to those published elsewhere $(18,22)$. However, the higher serum calcium and PTH values suggest more advanced disease in our cohort. The most common lesion was parathyroid adenoma, found in $92 \%$ of cases-a much higher rate than that previously reported $(78 \%)(18,22)$. This difference may be due to a selection bias, as our study covers a period of 18 years. At the beginning of this period, there was certain reluctance among Endocrinologists to indicate surgery in patients with negative imaging tests, a situation more frequently associated with MGD.

Studies reporting higher rates of MGD have tended to use a definition based on intraoperative findings such as the presence of enlarged glands. In most of these studies, bior unilateral neck exploration was performed, even in cases of preoperative workup suggestive of SGD. Some of them report the removal of additional slightly enlarged parathyroid glands, resulting in a diagnosis of MGD that is at least controversial $(17,22)$. Our definition of single-gland PHPT (a fall in ioPTH meeting Miami criteria) is more "functional", and is based on our experience using mainly focused surgery.

The etiology of MGD is multifactorial. It is frequently related to hereditary familial syndrome (33), which explains why the number of patients diagnosed with hereditary PHPT was significantly higher in patients with MGD.

In our study, the percentage of patients with a CaPTHUS score $\geq 3$ was substantially higher $(73.7 \%)$ than the rates published in the initial study (35\%) (18) and by the Wisconsin group (22\%) (19). This discrepancy may be due to the fact that the two populations (European and US) were diagnosed at different stages of the disease using different evaluation protocols. In the study by Elfenbein et al., the CaPTHUS score was applied to a population with a milder PHPT (22), in accordance with the current standard treatment of patients with asymptomatic PHPT $(34,35)$. In addition, that study included patients with missing preoperative imaging tests, who received a lower score.

In our cohort, the CaPTHUS model demonstrated an intermediate ability to detect SGD, with an area under the ROC curve of 0.74 . There were cases of MGD that met three or more factors, so if we rely on this model alone, 3.4\% of patients would not be adequately treated with selective surgery. To minimize this risk, we should continue to require perioperative adjuncts or carry out more unilateral or BME. There are even some groups that reject the unilateral selective approach and advocate BNE systematically (36).

In our study, we did not find patients with WIN values $<800$, as reported in the original study (19), probably because our patients presented more advanced stages of the disease. We found that a WIN greater than 2,000 and a gland weight above $1 \mathrm{~g}$ had a PPV of $92.5 \%$ for SGD, higher than the figures of $81 \%$ in the initial study (19) and $86.7 \%$ in the study by Edafe $e t$ al. (23). This guides us that in recent years 
patients with milder forms of PHPT were operated.

Our study has a number of limitations. Given its retrospective nature and the long inclusion period, there may be a selection bias, with respect to non-surgical patients not included in the study. The second limitation is its single-center design. Third, there may have been some observer bias, since the imaging tests and surgical techniques are operator-dependent; in addition, both were modified and improved during the study period.

Among the strengths of this research, we can include its focus on a closely-followed series, selected using strict exclusion criteria, treated at an established Endocrine Surgery Unit and maintaining the same criteria over time. The inconsistency of the results with respect to previous similar studies can be attributed to the more advanced stage of disease in our cohort; this became evident when analysing the CaPTHUS score and the WIN Index, which presented higher values than in previous studies. Regarding the severity, looking at the calcium and PTH figures, possibly more symptomatic patients with SGD have been operated on than in the USA or the UK. Reasons supporting this assumption includes a possible lesser detection of asymptomatic PHPT and some degree of rejection of endocrinologists to propose surgery to patients with negative workup. Furthermore, in our results the CaPTHUS model accurately predicted SGD in $96.6 \%$ of the patients, a percentage higher than the $91 \%$ described by Elfenbein et al. (22), but slightly lower than the $100 \%$ described by Kebebew et al. in the original work (18).

\section{Conclusions}

The CaPTHUS model was a useful tool for stratifying the suspicion of MGD as a cause of PHPT in a southern European population. However, having three or more predictors of SGD in the scoring model does not definitively rule out the possibility of MGD. For its part, the WIN did not allow us to establish a range of expected weights for parathyroid disease in every value of the product of calcium by PTH. Therefore, to minimize the risk of missing a MGD during selective surgery, we must continue to use supplementary intraoperative adjuncts such as ioPTH, or carry out bilateral explorations in cases with the slightest suspicion of additional parathyroid pathology.

\section{Acknowledgments}

The present work shows partial results of the doctoral thesis "Utility of the Wisconsin and CaPTHUS indices for the prediction of multiglandular disease in patients with primary hyperparathyroidism scheduled for selective parathyroidectomy" by Mario Serradilla-Martín at the University of Granada, Spain.

Funding: None.

\section{Footnote}

Reporting Checklist: The authors have completed the STARD reporting checklist. Available at http://dx.doi.org/10.21037/ gs-20-857

Data Sharing Statement: Available at http://dx.doi. org/10.21037/gs-20-857

Conflicts of Interest: All authors have completed the ICMJE uniform disclosure form (available at http://dx.doi. org/10.21037/gs-20-857). The authors have no conflicts of interest to declare.

Ethical Statement: The authors are accountable for all aspects of the work in ensuring that questions related to the accuracy or integrity of any part of the work are appropriately investigated and resolved. The study was conducted in accordance with the Declaration of Helsinki (as revised in 2013). Patients' informed consent was not required since this was a retrospective and observational study that did not entail any risk. The study was approved by the regional ethics committee of Granada (PI19/127).

Open Access Statement: This is an Open Access article distributed in accordance with the Creative Commons Attribution-NonCommercial-NoDerivs 4.0 International License (CC BY-NC-ND 4.0), which permits the noncommercial replication and distribution of the article with the strict proviso that no changes or edits are made and the original work is properly cited (including links to both the formal publication through the relevant DOI and the license). See: https://creativecommons.org/licenses/by-nc-nd/4.0/.

\section{References}

1. Mogollón-González M, Notario-Fernández P, Dominguez-Bastante $\mathrm{M}$, et al. The CaPTHUS score as predictor of multiglandular primary hyperparathyroidism in a European population. Langenbecks Arch Surg 2016;401:937-42. 
2. Barczyński M, Bränström R, Dionigi G, et al. Sporadic multiple parathyroid gland disease-A consensus report of the European Society of Endocrine Surgeons (ESES). Langenbecks Arch Surg 2015;400:887-905.

3. Thier M, Daudi S, Bergenfelz A, et al. Predictors of multiglandular disease in primary hyperparathyroidism. Langenbecks Arch Surg 2018;403:103-9.

4. Bilezikian JP, Brandi ML, Eastell R, et al. Guidelines for the management of asymptomatic primary hyperparathyroidism: summary statement from the Fourth International Workshop. J Clin Endocrinol Metab 2014;99:3561-9.

5. Lundgren E, Ridefelt P, Åkerström G, et al. Populationbased screening for primary hyperparathyroidism with serum calcium and parathyroid hormone values in menopausal women. Surgery 1997;121:287-94.

6. Adami S, Marcocci C, Gatti D. Epidemiology of primary hyperparathyroidism in Europe. J Bone Miner Res 2002;17 Suppl 2:N18-23.

7. Sackett WR, Barraclough B, Reeve TS, et al. Worldwide trends in the surgical treatment of primary hyperparathyroidism in the era of minimally invasive parathyroidectomy. Arch Surg 2002;137:1055-9.

8. Fraser WD. Hyperparathyroidism. Lancet 2009;374:145-58.

9. Soyder A, Ünübol M, Ömürlü IK, et al. Minimally invasive parathyroidectomy without using intraoperative parathyroid hormone monitoring or gamma probe. Ulus Cerrahi Derg 2015;31:9-14.

10. Sung JY. Parathyroid ultrasonography: the evolving role of the radiologist. Ultrasonography 2015;34:268-74.

11. Press DM, Siperstein AE, Berber E, et al. The prevalence of undiagnosed and unrecognized primary hyperparathyroidism: a population-based analysis from the electronic medical record. Surgery 2013;154:1232-7.

12. Goldstein RE, Blevins L, Delbeke D, et al. Effect of minimally invasive radioguided parathyroidectomy on efficacy, length of stay, and costs in the management of primary hyperparathyroidism. Ann Surg 2000;231:732-42.

13. Udelsman R. Six hundred fifty-six consecutive explorations for primary hyperparathyroidism. Ann Surg 2002;235:665-70.

14. Chen H, Mack E, Starling JR. A compressive evaluation of perioperative adjuncts during minimally invasive parathyroidectomy: which is most reliable? Ann Surg 2005;242:375-80.

15. Haciyanli M, Lal G, Morita E, et al. Accuracy of preoperative localization studies and intraoperative parathyroid hormone assay in patients with primary hyperparathyroidism and double adenoma. J Am Coll Surg 2003;197:739-46.

16. Carneiro DM, Solorzano CC, Nader MC, et al. Comparison of intraoperative iPTH assay (QPTH) criteria in guiding parathyroidectomy: which criterion is the most accurate? Surgery 2003;134:973-9.

17. Kavanagh DO, Fitzpatrick P, Myers E, et al. A predictive model of suitability for minimally invasive parathyroid surgery in the treatment of primary hyperparathyroidism. World J Surg 2012;36:1175-81.

18. Kebebew E, Hwang J, Reiff E, et al. Predictors of singlegland vs. multigland parathyroid disease in primary hyperparathyroidism: a simple and accurate scoring model. Arch Surg 2006;141:777-82.

19. Mazeh H, Chen H, Leverson G, et al. Creation of a "Wisconsin index" nomogram to predict the likelihood of additional hyperfunctioning parathyroid glands during parathyroidectomy. Ann Surg 2013;257:138-41.

20. Solorzano CC, Carneiro-Pla D. Minimizing cost and maximizing success in the preoperative localization strategy for primary hyperparathyroidism. Surg Clin North Am 2014;94:587-605.

21. Tublin ME, Pryma DA, Yim JH, et al. Localization of parathyroid adenomas by sonography and technetium Tc 99m sestamibi single-photon emission computed tomography before minimally invasive parathyroidectomy: are both studies really needed? J Ultrasound Med 2009;28:183-90.

22. Elfenbein DM, Weber S, Shneider DF, et al. CaPTHUS scoring model in primary hyperparathyroidism: can it eliminate the need for ioPTH testing? Ann Surg Oncol 2015;22:1191-5.

23. Edafe O, Collins EE, Ubhi CS, et al. Current predictive models do not accurately differentiate between single and multi gland disease in primary hyperparathyroidism: a retrospective cohort study of two endocrine surgery units. Ann R Coll Surg Engl 2018;100:140-5.

24. Sepahdari AR, Bahl M, Harari A, et al. Predictors of Multigland Disease In Primary Hyperparathyroidism: A Scoring System with 4D-CT imaging and biochemical markers. AJNR Am J Neuroradiol 2015;36:987-92.

25. Rodgers SE, Hunter GJ, Hamberg LM, et al. Improved preoperative planning for directed parathyroidectomy with 4-dimensional computed tomography. Surgery 2006;140:932-40.

26. Cheung K, Wang TS, Farrokhyar F, et al. A meta-analysis of preoperative localization techniques for patients with primary hyperparathyroidism. Ann Surg Oncol 
2012;19:577-83.

27. Kukar M, Platz TA, Schaffner TJ, et al. The use of modified fourdimensional computed tomography in patients with primary hyperparathyroidism: an argument for the abandonment of routine sestamibi single-positron emission computed tomography (SPECT). Ann Surg Oncol 2015;22:139-45.

28. Sho S, Yilma M, Yeh MW, et al. Prospective Validation of Two 4D-CT-Based Scoring Systems for Prediction of Multigland Disease in Primary Hyperparathyroidism. AJNR Am J Neuroradiol 2016;37:2323-7.

29. Ballester Vázquez E, Pérez García JI, López Mora DA, et al. Identification of Occult Adenomas in Primary Hyperparathyroidism With 18F-fluorocholine PET/CT. Cir Esp 2020;98:395-402.

30. Treglia G, Piccardo A, Imperiale A, et al. Diagnostic performance of choline PET for detection of hyperfunctioning parathyroid glands in hyperparathyroidism: a systematic review and metaanalysis. Eur J Nucl Med Mol Imaging 2019;46:751-765.

31. Broos WAM, van der Zant FM, Knol RJJ, et al. Choline PET/CT in parathyroid imaging: a systematic review.

Cite this article as: Serradilla-Martín M, Palomares-Cano A, Cantalejo-Díaz M, Mogollón-González M, Brea-Gómez E, Muñoz-Pérez NV, Arcelus-Martínez JI, Villar-del-Moral JM. Usefulness of the Wisconsin and CaPTHUS indices for predicting multiglandular disease in patients with primary hyperparathyroidism in a southern European population. Gland Surg 2021;10(3):861-869. doi: 10.21037/gs-20-857
Nucl Med Commun 2019;40:96-105.

32. Evangelista L, Ravelli I, Magnani F, et al. 18F-choline PET/CT and PET/MRI in primary and recurrent hyperparathyroidism: a systematic review of the literature. Ann Nucl Med 2020;34:601-19.

33. Al-Salameh A, Baudry C, Cohen R. Update on multiple endocrine neoplasia Type 1 and 2. Presse Med 2018;47:722-31.

34. AACE/AAES Task Force on Primary Hyperparathyroidism. The American Association of Clinical Endocrinologists and the American Association of Endocrine Surgeons position statement on the diagnosis and management of primary hyperparathyroidism. Endocr Pract 2005;11:49-54.

35. Wilhelm SM, Wang TS, Ruan DT, et al. The American Association of Endocrine Surgeons Guidelines for Definitive Management of Primary Hyperparathyroidism. JAMA Surg 2016;151:959-68.

36. Norman J, Lopez J, Politz D. Abandoning unilateral parathyroidectomy: why we reversed our position after 15,000 parathyroid operations. J Am Coll Surg 2012;214:260-9. 